\title{
Novel Method using Beacon and Smart Phone for Visually Impaired/Blind People
}

\author{
Ayad E. Korial \\ Department of computer engineering, \\ University of Technology, Baghdad, Iraq
}

\author{
Mohammed N. Abdullah \\ Department of computer engineering, \\ University of Technology, Baghdad, Iraq
}

\begin{abstract}
This paper presents novel structure for visually impaired/blind people using beacon and smart phone. The proposed structure is consisted of three parts. In the first part esp8266 module due to ultra-low power consumption, in the second part configurator application to configure these beacon and last part is mobile application to detect these beacons. The aim is to help visually impaired/blind people to knowledge the environment in which they live by. Three tests applied in real environment. The results show good performance for the suggested scheme help the visually impaired/blind people reach the desired devices location successfully without error. In conclusion, beacon and smart phone were a valid and reliable method to help the visually impaired/blind people to know the location of devices that are nearest from him in indoor environment.
\end{abstract}

\section{General Terms}

Beacon, nearby notification.

\section{Keywords}

Beacons, smart phone, visually impaired/blind people, RSSI, esp8266 module and indoor navigation.

\section{INTRODUCTION}

One of many supports that is urgent is the guarantee of mobility for blind people. There has been many efforts but even now, it is not easy for blind people to independently move [1].

Sentient computing is the proposition that applications can be made more responsive and useful by observing and reacting to the physical world. It is particularly attractive in a world of mobile users and ubiquitous computers [2].

Blind and visually impaired people have different important daily needs including navigation. Navigation and way finding is crucial for the blind population, as for any other. Visually impaired people in new environments may feel totally disoriented or isolated. These people can easily end up in dangerous and confusing situations as they move in unknown places [3].

Visually impaired people rely on different tools and skills to navigate. They usually rely on their white canes, seeing-eyedogs and other skills acquired over time to aid their navigation. Visually impaired people, that are able to perceive sounds, tend to learn how to identify audible landmarks. In general, people supplement their loss of sight by becoming better with other senses even smell [4].

Positioning objects has been an important topic since it is needed to locate people, guide them to a certain place, and assist companies and organizations with their assets management [5].

Due to the large number of applications that can benefit from a location service in indoor environments, indoor location systems have been an important research topic in recent years. Because of the advances in wireless technologies and the consequent proliferation of wireless devices in indoor buildings, the use of radio frequency signals to perform localization has become an interesting and promising technique to build better location systems [6].

Tsung et al., (2009) [7] presented an experimental study, in which they deploy a 14-node 802.15.4-based sensor network in them department building. Using the k-nearest-neighbor (KNN) method to the estimation of location, they implement an RSSI-signature-based localization system on top of the network.

Kajioka et al., (2014) [8] suggested real-time indoor location estimation needs multiple signals from different sources. To ensure accuracy of estimation, adopting Bluetooth Low Energy (LE) beacon devices as signal sources is a reasonable solution. They have installed a number of Bluetooth Low Energy (LE) beacon devices in and around a room and observed beacons by portable devices with RSSI to achieve fundamental knowledge of beacons and feasibility of location estimation. By applying an estimation method, they have achieved over $95 \%$ of correct estimation rate and complete separation that a device was in a room or not.

$\mathrm{Li}$ et al., (2014) [9] proposed a passive Wi-Fi source localization system based on fine-grained power-based trilateration. A Wi-Fi-based passive localization system can provide user location information to third-party providers of positioning services. However, indoor localization techniques are prone to multipath and Non-Line Of Sight (NLOS) propagation, which lead to significant performance degradation. To overcome these problems, they provided a passive localization system for Wi-Fi targets with several improved algorithms for localization.

Rida et al., (2015) [10] described indoor location position based on Bluetooth signal strength. Nowadays many systems with diverse technologies such as (GPS, Wi Fi, Bluetooth, ZigBee, Ultra-Wide Band, Ultrasounds, Infrared, etc...) can be used for indoor location. One of the biggest challenges in indoor location systems is to determine the actual indoor position of the user using a smart device (Phone, PC, etc...) due to their instability, cost, high-power consumption, low accuracy, and low precision. To overcome those problems, they have designed, implemented and emulated an indoor location system based on RSSI (Receive Signal Strength indicator) of the Bluetooth low energy 4.0 (BLE). BLE 4.0 technology is more advantageous in terms of long life expectancy; the approach was based on the deployment of equidistant nodes on the ceiling. The nodes are programmed to broadcast a periodic beacon at a time space of $400 \mathrm{~ms}$ and then to enter sleep mode. When a smart device enters within the broadcasting range, it locates the three access points (nodes) with the highest signal strength. This information calculated using one of the several localization algorithms (Trilateration) .The proposed Trilateration algorithm can be 
easily implemented in the hardware due to its low complexity; the method can be successful even when using a few numbers of nodes. In average, the system has about $0.5 \sim 1$ meters of error.

In this paper, novel method using beacon and smart phone for visually impaired/blind people is presented operating in more than one run, using $\mathrm{C}$ sharp and Java languages for smart phone.

\section{SYSTEM DESIGN}

\subsection{Configurator of the system}

The configuration of beacons need two things, application to configure the beacon and the circuit to connect the beacon with the application. Fig 1 shows the configurator application programmed using $\mathrm{C} \#$ and configurator circuit.

This application can connect serially with the beacon via configurator circuit by determining the PORT and the BAUD rate of the chip. Determination both previous things then press connect button to start connecting the application with the beacon. After that, the monitor part shows AT OK message if successfully connected.

Finally, need to enter the beacon name and press the button change to change the name of it. After all previous things, press disconnect button and then remove the beacon chip from the configurator circuit and can used it individually. Our configurator circuit contains the module of the "Future Technology Devices International" commonly known by its abbreviation FTDI. This module is specialized in universal serial bus USB that allows us to convert the USB port to serial with all port pins such as TX, RX, CTS, DTR, GND and VCC. The ESP8266 can interface with FTDI using socket $4 \times 2$, and also putting the jumper connector can easily switch between normal mode and firmware download mode.

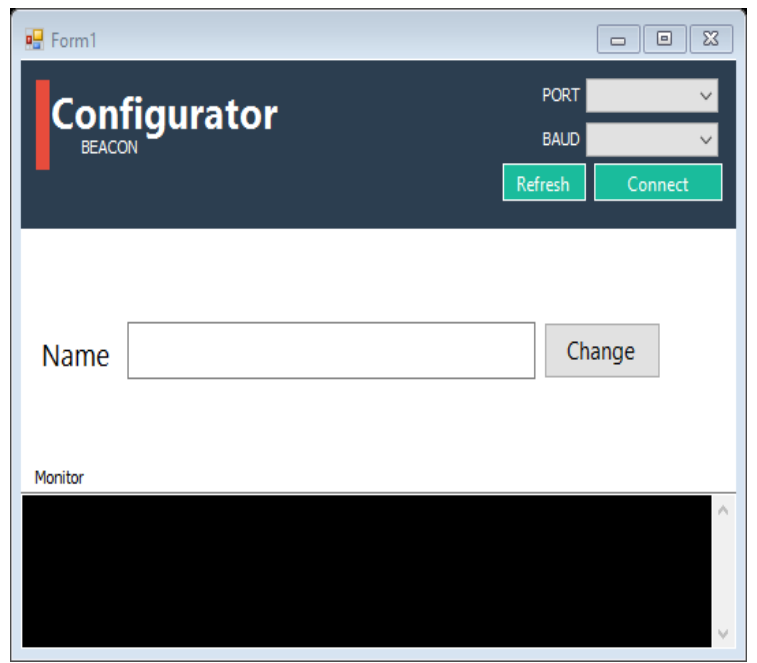

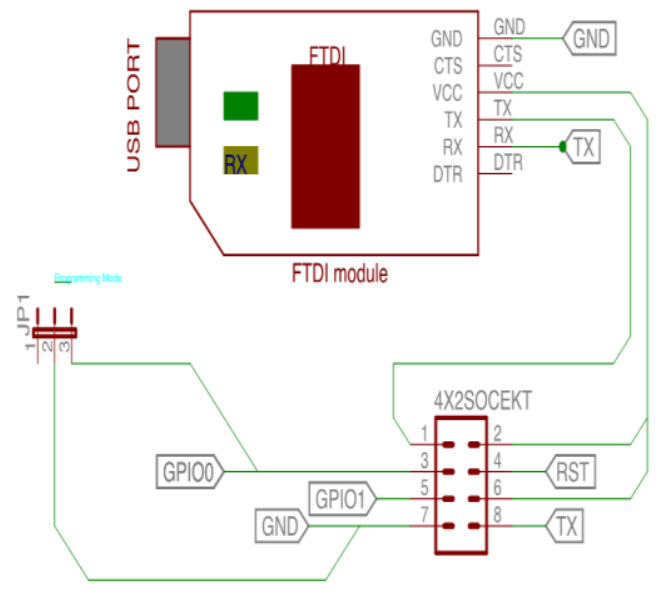

Fig 1: The configurator application and configurator circuit

\subsection{Beacon electronic circuit}

Beacon circuit contains the ESP8266 with power supply and voltage regulator to regulate the voltage of the power supply to 3.3 volt that is the same voltage of the ESP8266.

To enable the chip, pull CH_PD HIGH with a resistor to $+3.3 \mathrm{v}$ as shown in Fig 2.

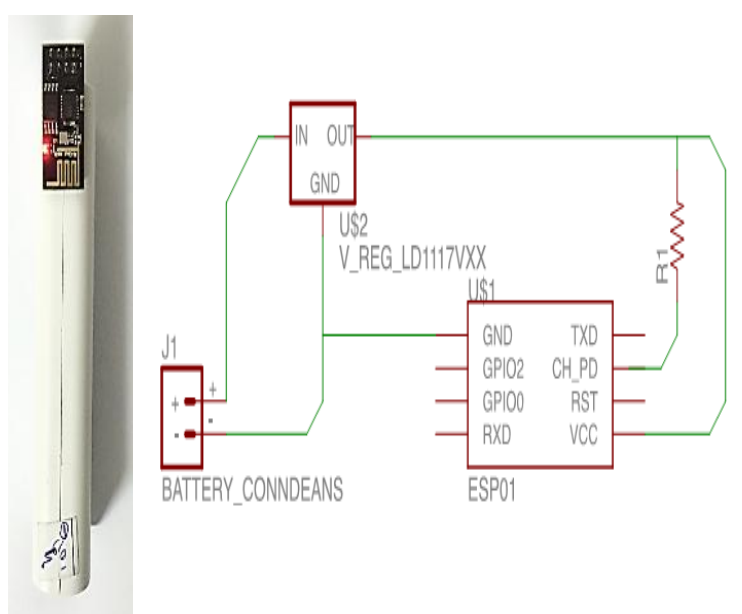

Fig 2: Beacon main circuit

\subsubsection{Add beacon to mobile}

To add one or more beacon to the database of the application, by opening our mobile application and going to nearby activity then pressing the plus sign "add button" this action can take us to new activity, scan for beacon activity, the mobile start to scan for near bacon.

If it detected beacon, then mobile will directly show the information of that beacon such as name and mac address of the chip with green image, and then press add button to save it in application to use these information later.

If mobile did not detect beacon, then it will show null information and with red image. Fig 3 show the process to add beacon to mobile.

Hence received signal strength is related to distance using the equation 1 below.

$R S S I[d B m]=-10 n \log _{10}(d)+R S S I_{1 m}[d B m]$

Where $\mathrm{n}$ is the propagation path loss exponent, $\mathrm{d}$ is the distance from the sender and $\mathrm{A}$ is the received signal strength 
at one meter of distance.

Simplify equation (1), obtain equation (2) below:

$d=10\left(\frac{R S S I[\mathrm{dBm}]-R S S I 1 m[\mathrm{dBm}]}{-10 n}\right)$
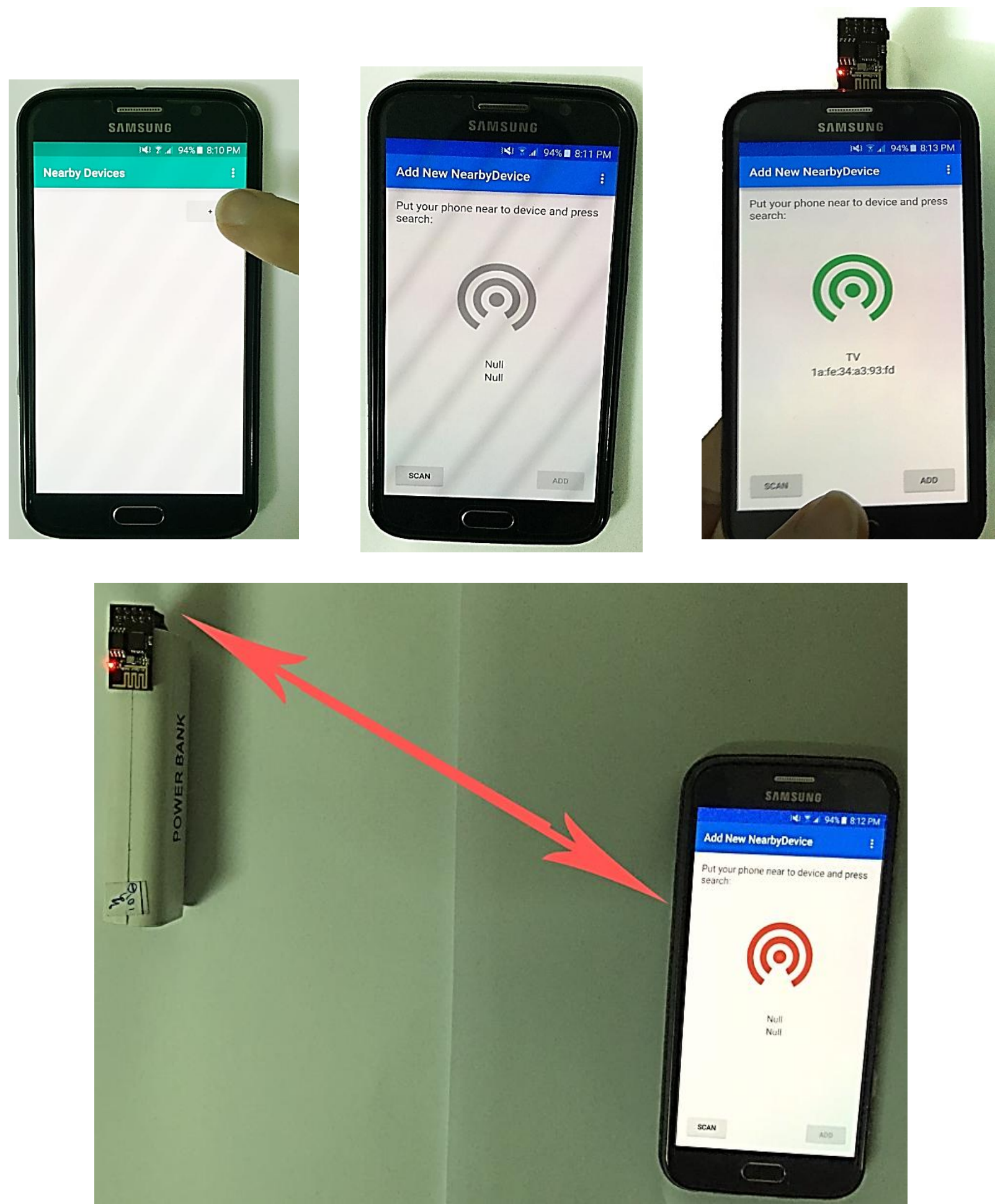

Fig 3: Add beacon to mobile

Fig 4 shows Add beacon to mobile flow chart 


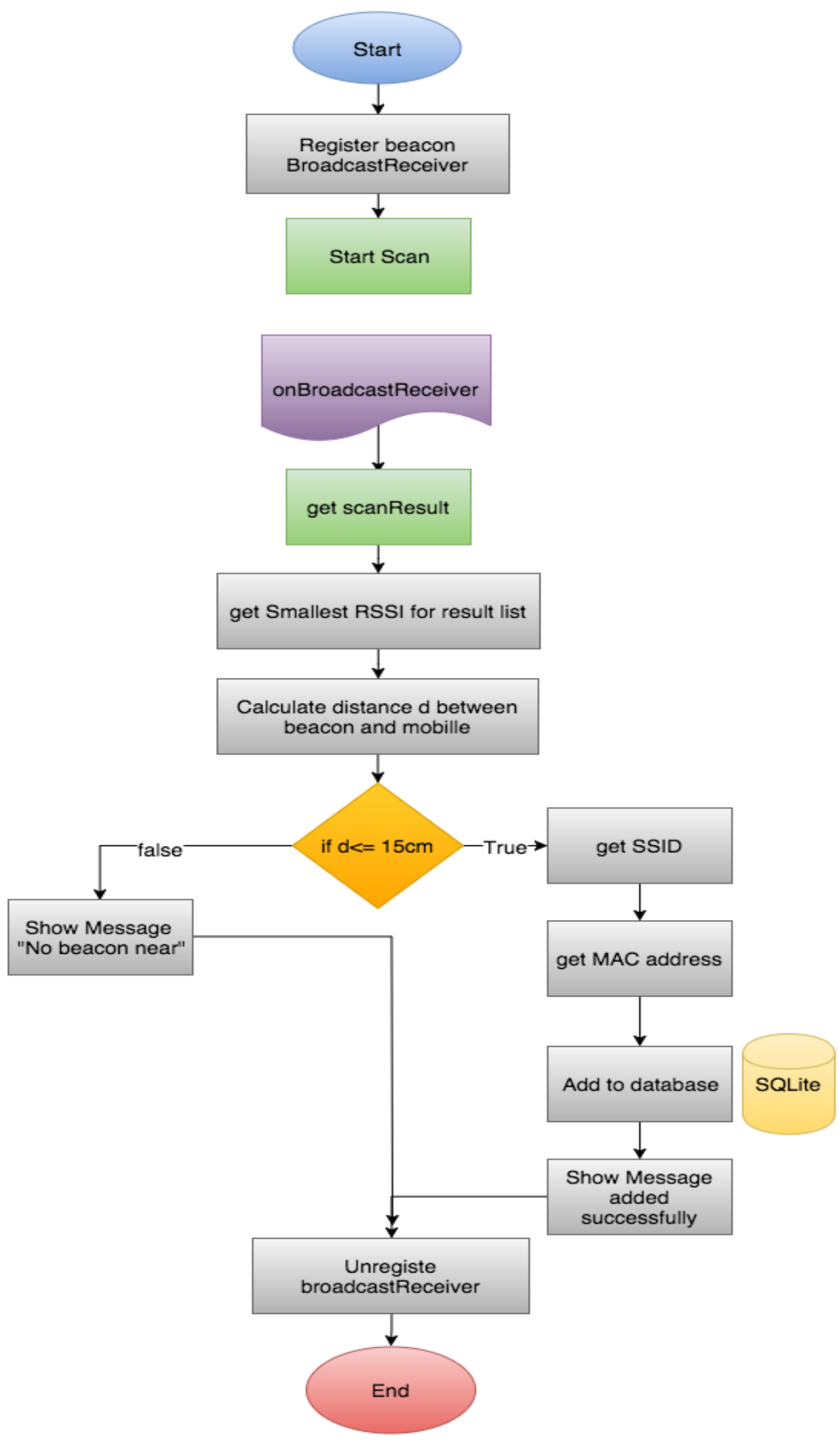

Fig 4: Add beacon to mobile flow chart

2.2.2 Nearby notification flowchart

Fig 5 shows nearby notification 


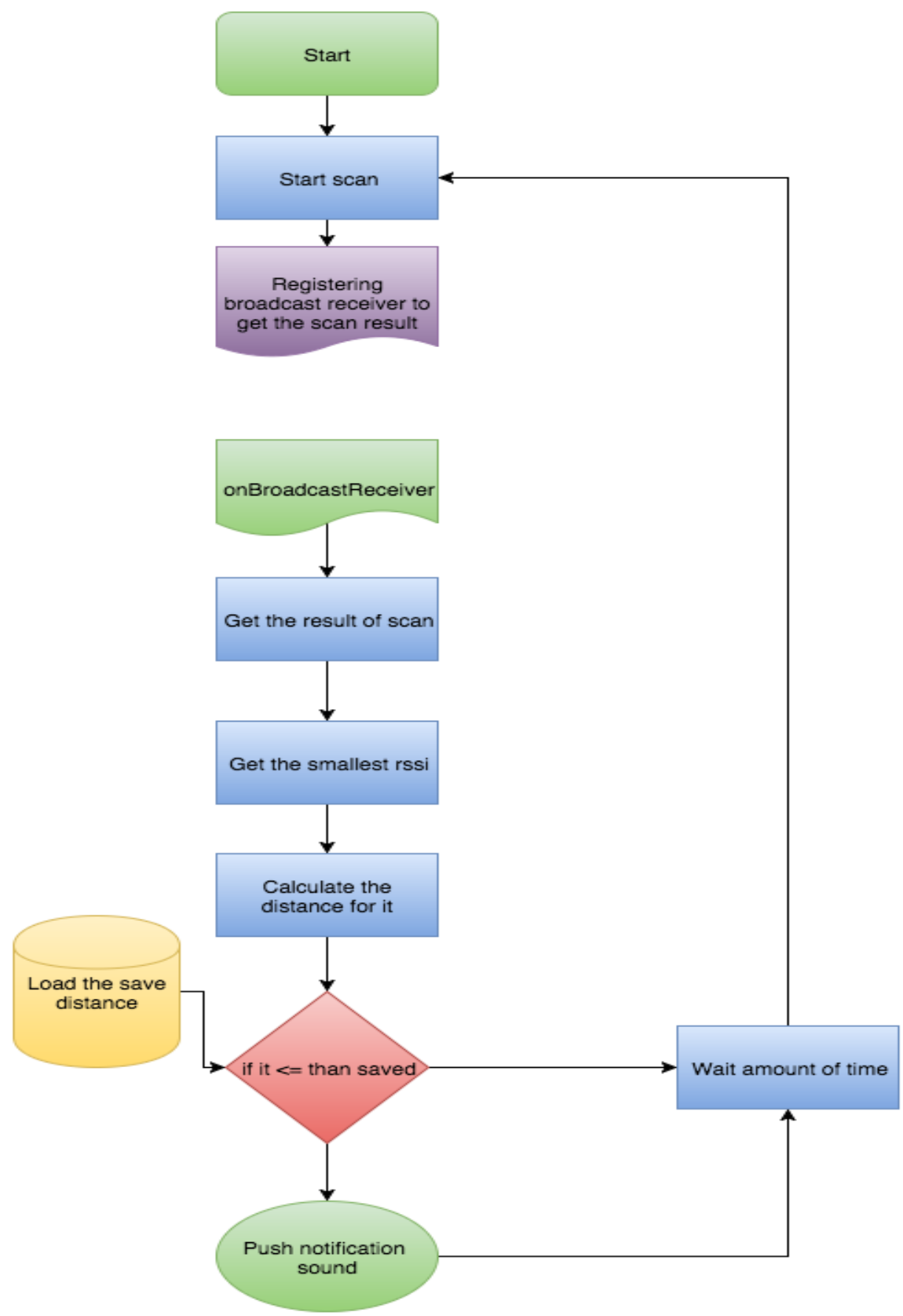

Fig 5: Nearby notification flow chart

\section{RESULTS AND DISCUSSION}

The test can be done by putting some beacons in different places in indoor, such as, the first, put on the Television and the second beacon put on refrigerator and the last one put on the water dispenser as an example.

Mobile detect the near beacon with short distance that put in setting of the application. If the mobile put between two or more beacon, mobile calculate the distance from all beacon and still choose the nearest of it.

Below Fig 6 shows the results of nearby notification with different devices.

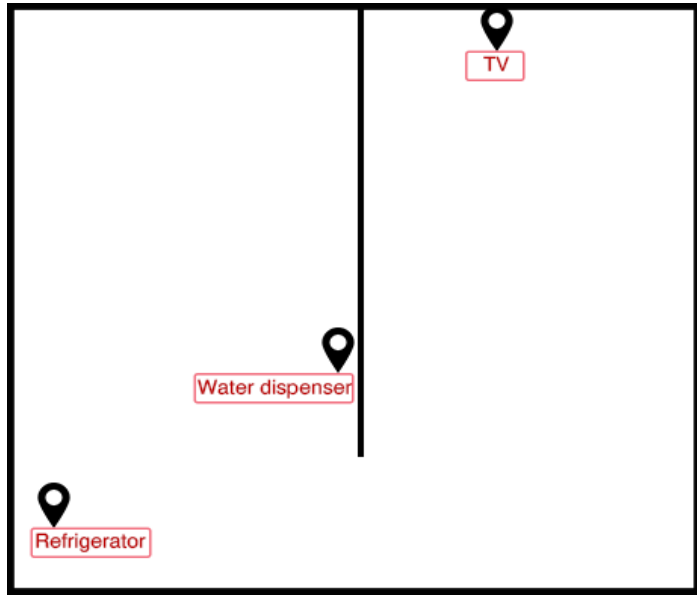

(a) 
The mobile calculates the distance of beacons and pushes sound notification in the case if it is near form them. When smart phone in the left room with location shown in Fig 6 b, In this case, mobile don't push notification because distance is large from all beacons.

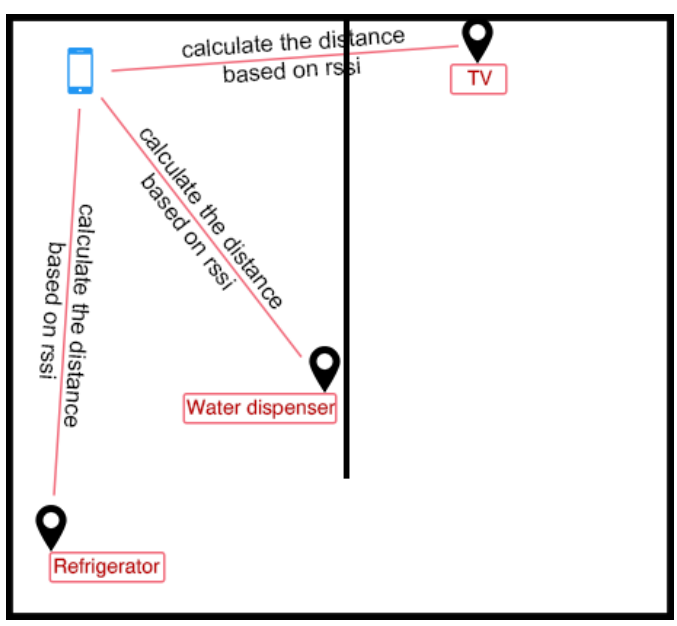

(b)

Mobile still in the left room but moved from previous location to the location shown in Fig $6 \mathrm{c}$ and the mobile calculates the distance of beacons and pushes sound notification in the case if it near form them. In this case mobile detects that it is near to the water disperser, as a result, the mobile pushes sound notification "You are nearby water dispenser".

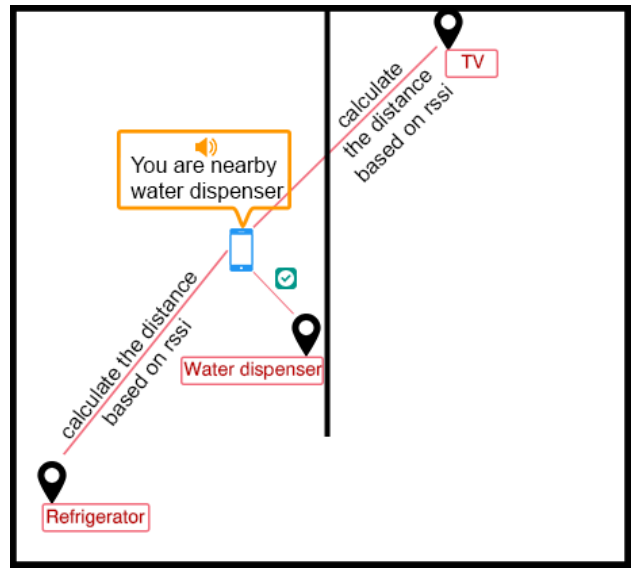

(c)

When mobile in the left room moved from previous location to location shown in Fig $6 \mathrm{~d}$ and still in the left room, then the mobile calculates the distance of beacons and pushes sound notification in the case if it is near form them. In this case, mobile detects that it is near to the refrigerator, as a result, the mobile pushes sound notification "You are nearby refrigerator".

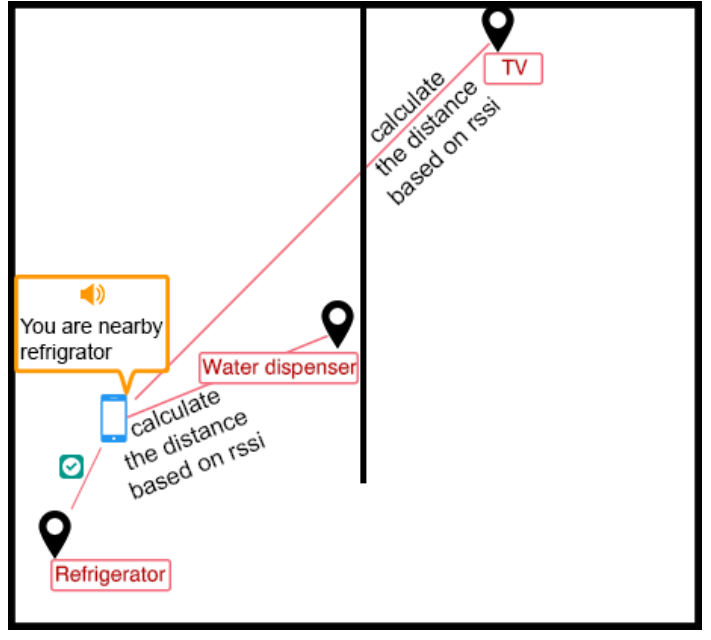

(d)

Finally, with the same of previous process and in case of right room, mobile detects that it is nearby the TV, as a result, the mobile pushes sound notification "You are nearby TV".

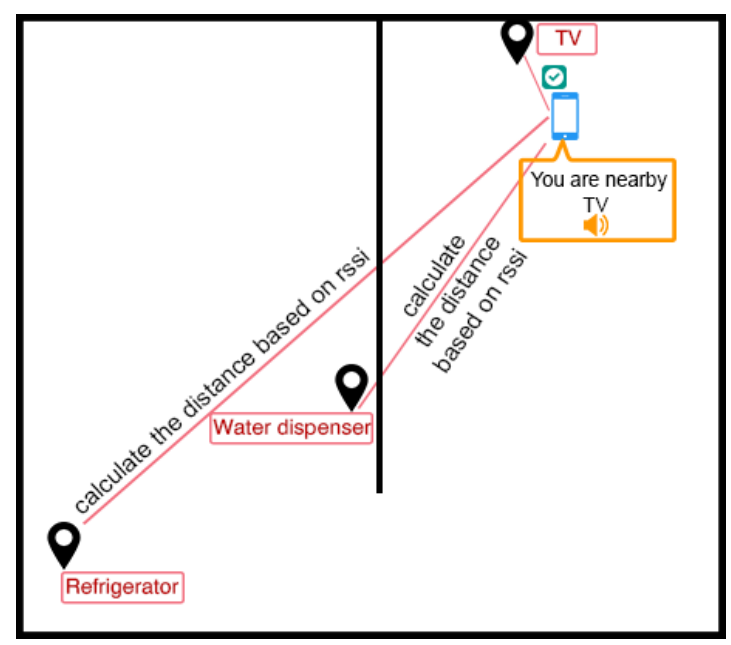

(e)

Fig 6: (a), (b), (c), (d) and (e) beacon nearby devices

\section{COMPARATIVE ANALYSIS BETWEEN THIS BEACON CHIP WITH OTHER CHIP}

Making a comparative analysis with the reference [11] according to points such as chip type, API, power consumption, distance notification, battery, blind usability and method to add it to mobile, and we found to the following:

\begin{tabular}{|c|c|c|}
\hline & This beacon & iHere tag [11] \\
\hline Chip & ESP8266 WIFI chip & Bluetooth chip \\
\hline API & $\begin{array}{c}\text { Easy to integration with AT } \\
\text { command }\end{array}$ & Not available \\
\hline Power \\
consumption & $\begin{array}{c}\text { Ultra-low power technology, } \\
\text { ESP8266 has been designed } \\
\text { for mobile, wearable } \\
\text { electronics and Internet of } \\
\text { Things applications } \\
\text { with the aim of achieving the } \\
\text { lowest power consumption }\end{array}$ & Low power \\
consumption \\
\multicolumn{2}{|c}{} \\
\hline
\end{tabular}




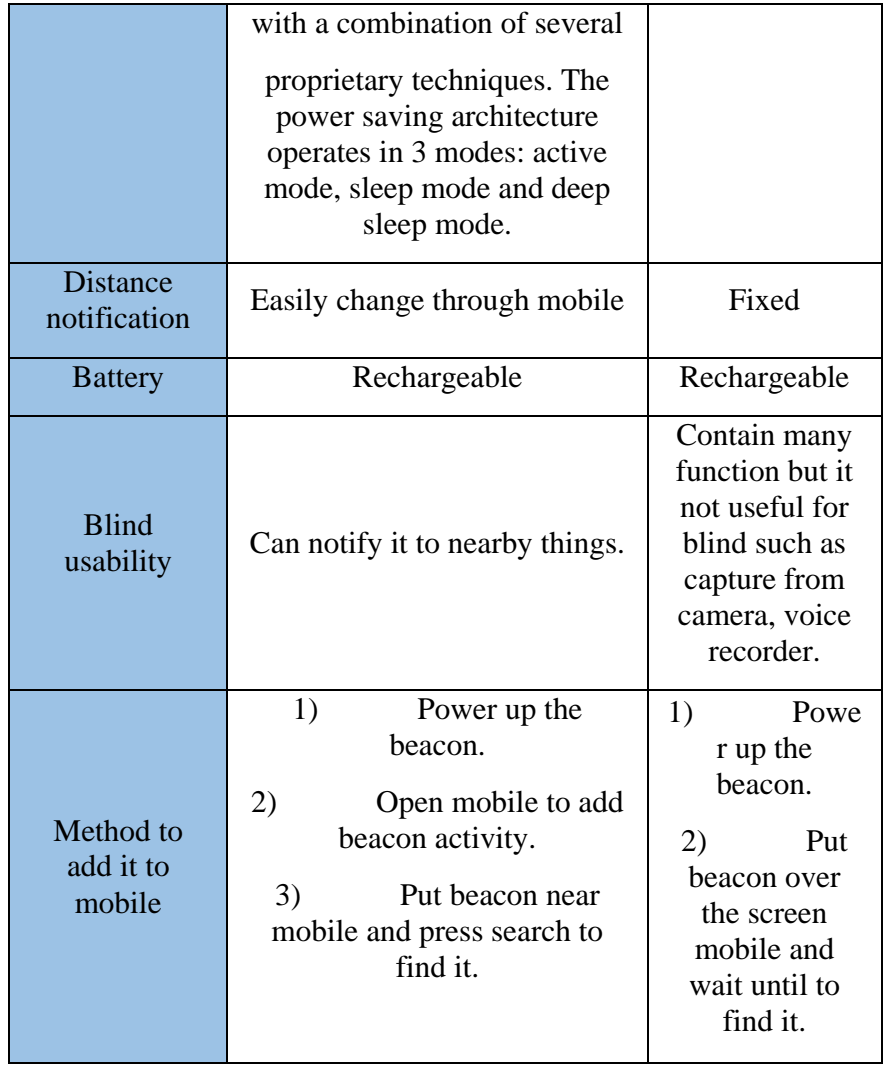

\section{CONCLUSION}

Nearby beacons where successfully and give responding from things. The results show good performance for the suggested scheme help the visually impaired/blind people reach the desired devices location successfully without error. Beacon and smart phone was a valid and reliable method to guide the visually impaired / blind people from a required device location to a desired device location in indoor environment. The suggestions for future work can be implement by using iPhone.

\section{REFERENCES}

[1] Kang C., Jo H. and Kim B., "A Machine-to-Machine based Intelligent Walking Assistance System for Visually Impaired Person", The Journal of KICS, Vol. 36, No. 3, 2011, pp. 195-304.
[2] Hopper A. "Sentient Computing", Phil. Trans. R. Soc. Lond., A, (2000), Vol. 358, pp. 2349-2358, August 2000.

[3] Hub A., Diepstraten J., Ertl T., "Design and development of an indoor navigation and object identification system for the blind". Assets '04 Proceedings of the 6th international ACM SIGACCESS conference on Computers and accessibility, pp. $147-152$, October 18 $-20,2004$.

[4] Gardner J., "Seeing with Other Senses: Gardens for the Blind". The Herb Companion, February 3, 2007. http://www.perkins.org/news-events/press/seeing-withother- senses.html, last visit November, 2015.

[5] Disha A.M. "A Comparative Analysis on indoor positioning Techniques and Systems", International Journal of Engineering Research and Applications, Vol. 3, Issue 2, March -April 2013, pp.1790.

[6] Liu H., Darabi H., Banerjee P., and Liu J., "Survey of Wireless Indoor Positioning Techniques and Systems," IEEE Trans. on Syst., Man, and Cybernetics, Vol. 37, No. 6, pp. 1067-1080, 2007.

[7] Tsung-Han Lin, I-Hei Ng, Seng-Yong Lau, Polly Huang, "Impact of Beacon Packet Losses to RSSI-SignatureBased Indoor Localization Sensor Networks". Mobile Data Management: Systems, Services and Middleware. MDM '09. Tenth International, pp. 389 - 390, 2009 1820 May 2009

[8] Kajioka, S., Mori, T. , Uchiya, T. , Takumi, I., "Experiment of indoor position presumption based on RSSI of Bluetooth LE beacon, Consumer Electronics (GCCE), 2014 IEEE 3rd Global Conference on, pp. 337 339, 7-10 Oct. 2014.

[9] Li Z., Braun T., and Dimitrova D.C.” A Passive WiFi Source Localization System based on Fine-grained Power-based Trilateration" World of Wireless, Mobile and Multimedia Networks (WoWMoM), 2015 IEEE 16th International Symposium , pp. 1-2, 14-17 June 2015.

[10] Rida M.E., Liu F., Jadi, Y., Algawhari A.A.A., Askourih A., "Indoor location position based on Bluetooth Signal Strength" Information Science and Control Engineering (ICISCE), 2015 2nd International Conference, pp. 769-770, 24-26 April 2015.

[11] http://www.nonda.co/products/ihere-rechargeablebluetooth-key-finder-remote-control-camera-shutter. Accessed on 3/8/2015. 